Research Article

\title{
Human Resources Balanced Allocation Method Based on Deep Learning Algorithm
}

\author{
Weiwei Shi $\mathbb{D I D}^{1,2}$ and Qiuzuo $\mathrm{Li}^{1}$ \\ ${ }^{1}$ PowerChina Henan Engineering Co., Ltd., Zhengzhou 450000, China \\ ${ }^{2} 58$.Com Inc., Beijing 100000, China \\ Correspondence should be addressed to Weiwei Shi; 20131011@stu.nmu.edu.cn
}

Received 8 August 2021; Revised 8 September 2021; Accepted 11 September 2021; Published 23 September 2021

Academic Editor: Muhammad Usman

Copyright (c) 2021 Weiwei Shi and Qiuzuo Li. This is an open access article distributed under the Creative Commons Attribution License, which permits unrestricted use, distribution, and reproduction in any medium, provided the original work is properly cited.

\begin{abstract}
At present, the economics and social developments show the characteristics of diversification, and the focus of social enterprise management is driven by the allocation of human resources. Human resource allocation is a way of appropriate allocation and reasonable placement of human resources. It means that, under the guidance of science, human resources can maintain the best combination with other resources at any time. Nevertheless, the irregularities in management teams and the balanced differences of talent quality have a great effect on the balanced development of an enterprise. Based on this, this paper studies the establishment of a recurrent neural network (RNN) model to realize the allocation of human resources and the balanced development of enterprise management. Firstly, a deep learning model, based on the recurrent neural network, is established. Then, the human resources data is analyzed to calculate the matching degree between the human resources and posts. Finally, personnel scheduling is carried out according to the matching degree score between the human resources and posts, to obtain the optimal balanced allocation result of the human resources. Experimental results show that our method can bring significant improvements to personnel position matching and effectively enhance the efficiency of human resource allocation based on the cloud environment.
\end{abstract}

\section{Introduction}

The term "human resource" refers to the sum of the labor capacity of workers in a specific field. There are many influencing factors on social productivity, and human resource is the most active and positive factor, which is the root of the existence and growth of a social organization [1]. In recent years, China's economy has gradually entered the "new normal." This is well known that advanced enterprise management methods and whether they can give full play to the value of employees have become important evaluation criteria for enterprise resource innovation and competitive advantage [2]. The human resource management personnel of the engineering unit shall analyze the ability of the unit's employees, allocate human resources reasonably, and form a working state of combining strength and weakness [3]. The optimization of human resource allocation is mainly divided into (i) establishing the idea of optimal allocation of human resources; (ii) updating the organizational structure; (iii) clarifying the position authority; (iv) integrating management methods; (v) providing effective guarantee for position management; and (vi) strengthening targeted training [4]. The formulation and implementation of human resource management policies are not effective, which has seriously affected the operation of modern enterprises. Therefore, the scientific and reasonable human resource allocation method is the sword of enterprise management.

At present, the computing models applied in the field of human resources are usually based on statistical algorithms, which cannot meet the processing needs of massive data and do not consider the characteristics of data hiding, which easily leads to the problem of lack of information [5]. Among the classical assignment algorithms, there is a quantitative assignment algorithm $[5,6]$. Individuals need to complete $M$ 
tasks $(n \neq m$ is allowed), establish a mathematical model based on the operational research method, and then obtain the assignment scheme based on the Hungarian method. However, the algorithm has some limitations when applied to practical work. This algorithm requires that the factors which determine the nature of different tasks are single and the same. If there are two or more different tasks in this $m$ task and the influencing factors are not single, then the efficiency index we need cannot be established through this algorithm. Therefore, the application of this algorithm in human resource allocation cannot be popularized [6]. As a result, this paper comprehensively considers several cases and selects the recurrent neural network algorithm to reasonably allocate human resources, that is, calculate and recommend human resources matching posts through the algorithm.

To improve the human resource system, this paper mainly solves the matching problem between the human resources and posts. Therefore, the input of the recommendation algorithm is the personal identity information of human resources, and the output is the position suitable for their identity. The dataset of the model contains numerous parameterized information such as human gender, personality, and professional qualifications, and the mapping dataset contains the parameterized information of the corresponding position. The human resource allocation problem to be solved in this paper is simplified to the problem of taking the given parameterized manpower as the input and calculating the predicted output through the algorithm. The major contributions of our work are as follows:

(i) We propose a new method to establish a recurrent neural network (RNN) to identify the allocation of human resources

(ii) A deep learning model based on a recurrent neural network is designed; then the human resources data are analyzed to calculate the matching degree between human resources and posts

(iii) Finally, the personnel scheduling is carried out according to the matching degree score between human resources and posts

The remainder of this paper is organized as follows: in Section 2, we introduce the related work. In Section 3, we introduce the methodology of our proposed model. In Section 4, we conduct experiments on a real-world dataset and compare the outcomes with some existing methods. Finally, we conclude the paper in Section 5 and offer several directions for future research.

\section{Related Work}

Human resource allocation is the corresponding recommendation and mapping between the human resources and posts. The application of the suggested recommendation algorithm can solve the problems described in this paper. A reasonable and applicable recommendation algorithm could potentially improve the efficiency of the balanced allocation of human resources. In recent years, recommendation algorithms have been widely used in many Internet enterprises. A complex and effective recommendation algorithm can mine users' multimodal information through users' history records and platform information, analyze users' various characteristics, predict people's preferences, accurately and intuitively analyze users' preferences, and push appropriate information to the users.

Compared with the existing traditional recommendation algorithms, deep learning series algorithms have strong advantages in the automatic extraction of abstract features. In recent years, with the rapid development of human electronic information technology, big data processing has been greatly improved, which also makes deep learning development rapidly and widely used in various domains [7]. In the following years, a series of deep learning algorithms have sprung up one after another. In 2006, based on the existing deep learning theory, Hinton et al. of the University of Toronto successively studied the relevant theories of Boltzmann machine and neural network [8]. The deep learning algorithm proposed by their team has achieved good results in terms of data classification and prediction. Then, in 2007, the authors in [9], developed a collaborative filtering algorithm based on the restricted Boltzmann machine (RBM), which is a generative stochastic neural network to train the scoring matrix of the visible layer data. Then, the trained model is used to predict users' preferences. At that time, SVD + + was the core of a series of deep learning models [10]. Due to its limitations, the series of algorithms almost fell into a deadlock. It was not until the emergence of RBM that the situation improved. When RBM appeared, the authors improved this series of depth algorithms and proposed RBM as a comprehensive generalization of the deep Boltzmann machine (DBM) [11], which is the first real depth learning model. Then, Netflix researchers studied the combination of GPU and AWS and introduced the distributed neural network theory [12]. After that, Spotify music company applied it to the music style analysis of users and made personalized recommendations according to the user's preferred song style, which once caused great repercussions [13]. In the deep learning model, the historical records of platform users can be regarded as a periodic series of data $[14,15]$. To analyze these time series data, various methods based on recurrent neural network (RNN) are preferred in the existing state-of-the-art literature [16]. As the founder of this usage, Netflix applied RNN to video recommendation systems, which promoted the research of deep learning algorithms of other companies and achieved significantly good and outstanding results.

Through the above research on the current situation at home and abroad, it can be concluded that the deep learning and recommendation algorithms have a mature development path [17]. After several updates and iterations, their applications have been widely used in both video and music recommendation, better match the user and system data, and recommend a better individual matching mode as compared to what was already available in the market. Similarly, applying this recommendation algorithm to job recommendations can complete the systematic, reasonable, and unbiased deployment of human resources. In this paper, 
the recurrent neural network model is used to learn the post manpower matching set and obtain a deeper post preference representation containing more useful information. This information can be used for the subsequent recommended posts and manpower matching.

\section{The Proposed Methodology}

According to the traditional human resource allocation theory, planning human resources is mainly to analyze the personnel structure of the unit, and sort out the relationship between post demand and personnel ability in detail. Personnel ability includes many elements. These elements are weighted and summed to judge the quality score of the personnel. The traditional human resource (HR) scoring process is shown in Figure 1.

Firstly, the received data is grouped and analyzed. The data grouping includes the personnel evaluation matrix and personnel capability matrix. The most widely used is to establish an employee ability evaluation matrix, which may consider many factors, such as self-evaluation, superior and subordinate evaluation, and patient evaluation. The personnel capability matrix includes employee performance, attendance rate, professional title, and other information. After the personnel evaluation matrix value and personnel capability matrix values are obtained, the key manpower and position matching degree can be obtained by the formula as illustrated in the following:

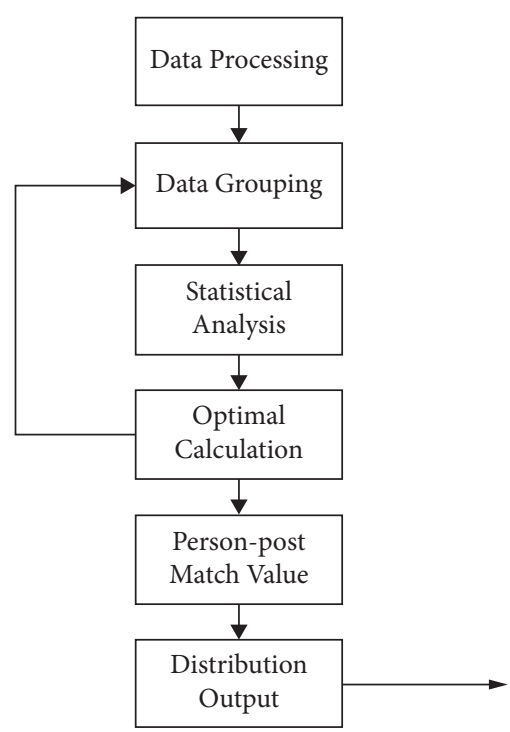

Figure 1: The human resources matching process.

$$
\left(H_{i j}\right)_{p \times q}=\left(n_{1}\left(a_{i j}\right)_{p \times 1}+n_{2}\left(b_{i j}\right)_{p \times 1}+\cdots+n_{4}\left(d_{i j}\right)_{p \times 1}\right),
$$

where $n_{1} \sim n_{4}$ is the corresponding evaluation parameter. Let another variable be $X_{i j}$, we have

$$
X_{i j}= \begin{cases}1, & \text { arrange personnel to corresponding positions, } \\ 0, & \text { no personnel will be assigned to the corresponding positions. }\end{cases}
$$

Therefore, the personnel can be optimized by the manpower and position matching degree model:

$$
\begin{aligned}
\max (Z & \left.=\sum_{i=1}^{n} \sum_{j=1}^{n} s_{i j} X_{i j}\right), \\
\sum_{i=1}^{n} X_{i j} & =1, \quad i \text { and } j=1,2, \ldots, m, \\
\sum_{i=1}^{n} X_{i j} & \leq 1, \quad i \text { and } j=1,2, \ldots, n .
\end{aligned}
$$

The above algorithm is simple and effective and can better configure human resources, but it is only applicable to the case of less human data. With the increase of hospital system, human data is also increasing, and the problem has become complex. This method has low computational efficiency, cannot mine data well, and cannot manage human resources effectively.

It can be seen from Figure 1 that the essence of the human resource scheduling model is to analyze human resource data and calculate the matching degree between the human resources and positions. Afterward, personnel scheduling is carried out according to the matching degree score between the manpower and position, which can be abstracted into a recommendation model in essence. Recommendation models have been analyzed and verified in many fields. At present, the mainstream recommendation models use a recurrent neural network as a primary and most important data processing module [18].

It should be noted that RNN records different historical information of time series data through the cyclic structure in the model. Its structural characteristics lead to its great advantages in processing various time series data. Different from ordinary neural networks, the hidden layers of recurrent (cyclic) neural networks are connected; that is, the output at the current time is not only related to the current input but also related to the output of the hidden layer at the previous time. The RNN model is shown in Figure 2. Among them, vector $X$, vector $S$, and vector $O$ represent the input data, hidden layer value, and output data in turn. Similarly, $U$ and $V$ represent the weight matrix from the input layer to the hidden layer and the weight matrix from the hidden layer to the output layer in turn. The value $S$ of the hidden layer depends not only on the value of $X$ but also on the value of $S$ of the last hidden layer. The weight matrix $W$ is the last value $S$, as shown in Figure 2, and denotes the input of this time. 


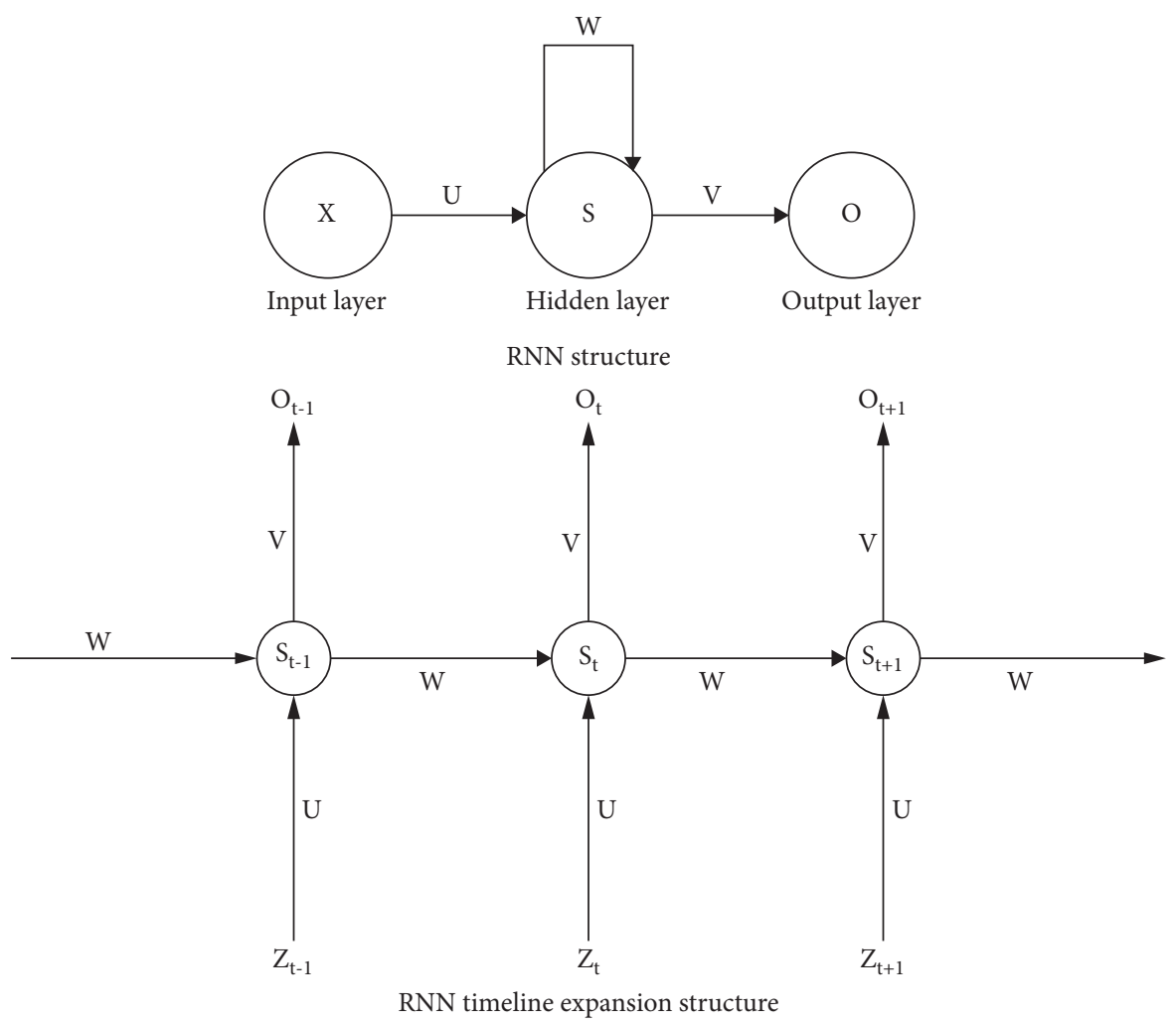

FIgURE 2: The RNN structure understanding diagram.

The network expanded in Figure 2 is the model at the time $t$, and the symbol correspondence is as follows: $z_{t}$ represents the input of input layer, $s_{t}$ represents the output of hidden layer, $g_{1}$ represents activation function of the output layer, $f_{1}$ represents activation function of the hidden layer, and $o_{t}$ represents the output of output layer. It is worth noting that the value of $s_{t}$ depends on $z_{t}$ as well as $s_{t-1}$. The forward calculation formula of the cyclic neural network is as follows:

$$
\begin{aligned}
o_{t} & =g_{1}\left(V s_{t}\right), \\
s_{t} & =f_{1}\left(U z_{t}+W s_{t-1}\right) .
\end{aligned}
$$

Equations (4) and (5) are used for cyclic iterative calculation, and the results are as follows:

$$
\begin{aligned}
o_{t} & =g_{1}\left(V s_{t}\right) \\
& =g_{1}\left(V f_{1}\left(U z_{t}+W s_{t-1}\right)\right) \\
& =g_{1}\left(V f_{1}\left(U z_{t}+W f_{1}\left(U z_{t-1}+W s_{t-2}\right)\right)\right) \\
& =g_{1}\left(V f_{1}\left(U z_{t}+W f_{1}\left(U z_{t-1}+W f_{1}\left(U z_{t-1}+\ldots\right)\right)\right)\right) .
\end{aligned}
$$

As can be seen from equation (6), the current time not only records the current time information but also contains historical information, which shows that the cyclic neural network has the function of memorizing historical information. However, the traditional cyclic neural network always calculates the state of the model by covering layer by layer. This method has some disadvantages. The chain rule of derivation is used for checking. This method directly leads to the function gradient expressed in the form of continuous multiplication, which makes the model have the fault problem of gradient disappearance.

The main feature of the recurrent neural network is to use cyclic convolution for data training operation. The cyclic convolution network model can be regarded as a hierarchical data model, and the input of the convolution network is the original human resource data. Through the processes of cyclic convolution, pooling, and activation function, the abstract features between data are extracted. The process expression is as follows:

$$
\begin{aligned}
& x^{1} \longrightarrow \omega^{1} \longrightarrow x^{2} \longrightarrow \cdots \longrightarrow x^{L-1} \longrightarrow \omega^{L-1} \\
& \quad \longrightarrow x^{L} \longrightarrow \omega^{L} \longrightarrow z, \\
& z=f\left(x^{L}, y\right)
\end{aligned}
$$

where $x^{L}$ is the data input of $L$ layer, $\omega$ is the parameter weight value of $L$ layer, $z$ is the loss function selected by the model, $y$ is the calibration value of the model, and $f$ is the final calculation parameter of the model. This paper improves the basic neural network, uses the hybrid cyclic neural network model, and uses the combination of global model and local model to take the data characteristics after hierarchical operation of the model as the network output, to realize the data processing. Then, the hierarchical model structure is used to build the network, to realize the matching recommendation of manpower and position. 
In the process of building the model, the cross entropy is selected as the judgment loss function. The loss function can compare the actual value of the data with the expected value of the data to determine the proximity of the data. The loss function is as follows:

$$
L=-\sum_{n}(y \log p+(1-y) \log (1-p)) .
$$

At the same time, the gradient optimization algorithm is used to optimize the parameters during training. In this way, the parameter transfer can be as accurate as possible. The specific update process of model parameters is as follows:

(1) Update the number of iterations and learning efficiency of the neural network:

$$
\begin{gathered}
m_{t}=\beta_{1} m_{t-1}+\left(1-\beta_{1}\right) g_{t}, \\
v_{t}=\beta_{2} v_{t-1}+\left(1-\beta_{2}\right) g_{t} .
\end{gathered}
$$

Among them, $\beta_{1}$ and $\beta_{2}$ are the hyperparameters of the cyclic neural network, $g_{t}$ is the calculation gradient of the model, and $t$ is the number of iterations of the model.

(2) Optimize the orientation of the first-order estimate and the second-order estimate, including

$$
\begin{gathered}
\widehat{m}_{t}=\frac{m_{t}}{1-\beta_{1}^{t}}, \\
\widehat{v}_{t}=\frac{v_{t}}{1-\beta_{2}^{t}} .
\end{gathered}
$$

(3) Update the parameters of the model from the results obtained above:

$$
\theta_{t+1}=\theta_{t}-\frac{l \widehat{m}_{t}}{\sqrt{\widehat{v}_{t}}+\varepsilon} .
$$

Combining the cyclic convolution neural network with the traditional human resource allocation algorithm, a human and post matching recommendation algorithm suitable for the field of human resources is designed in this paper. The algorithm not only improves the problem of low data training quality of traditional algorithms but also effectively improves the data calculation efficiency by using a cyclic neural network. The core idea of the algorithm can be elaborated in three major steps: (i) firstly, the original features in the data are extracted. The original features are consistent with the features required by traditional human resources, including personnel evaluation matrix and personnel ability matrix; (ii) then, the data is extracted to the encoder for coding; and (iii) finally the coding characteristics parameters are input to the input layer of the cyclic neural network. The cyclic convolution layer in the model is used to process the coded data, and then the matching results of manpower and position are obtained. The algorithm flow is shown in Figure 3.

Firstly, data acquisition is carried out, which uses the distributed flow acquisition method to select the data and group it into a personnel evaluation matrix and personnel ability matrix. Then, the data is abstracted, preprocessed, encoded by the encoder, and stored in a data warehouse. The lifting feature algorithm is used to further strengthen the data features and input to the cyclic convolution network. Finally, the matching score between the manpower and position is output, and the human resource recommendation process is completed.

The steps in the algorithm flow are described as follows: the first step is data collection and is categorized as follows:

(1) Data collection: The establishment of an employee position database is the basic work of constructing the deep learning model. Using modern information technology to establish an employee post database system can realize the automation of resource data parameter collection, processing, and management. It makes the statistical data information to steadily improve the results accuracy, preciseness, timeliness, and comprehensiveness. Employee position statistics is mainly used to collect, sort out, and store employee characteristic parameters and position characteristic parameters such as the number and age of various employees in the enterprise or among industries.

(2) Accuracy and timeliness of data: High accuracy employee post statistics can provide an effective guarantee for the human resource allocation system. At the same time, the statistical employee position data is timely, and the lagging database is difficult to match the enterprise information with a high iteration rate.

(3) Data integrity and pertinence: In the process of data collection, the comprehensiveness and integrity of data should be achieved. Based on this, we can get detailed and effective data information from the established database during query or statistics. In addition, whether the statistical data can bring value to decision-making depends on the rational use of the database and the availability of data. Therefore, when designing the database structure, we should consider that the data has the characteristics of comprehensiveness and pertinence, and the database has the characteristics of variable updatable, structure maintainable, fast update speed, and so on. This not only reduces the cost of data collection but also improves the effectiveness of data and effectively ensures the accuracy of the database.

(4) Continuity and nonsystematic structure of the data: In the process of employee position data statistics, we mainly pay attention to two dimensions: time and quantity. On the one hand, it stores the individual personnel status in the database. In addition to the existing status data, it also needs some historical data. These historical data can assist the system to complete the allocation of human resources. Based on the historical data of employees, they can deeply learn their personality characteristics. On the other hand, it saves the basic information of employees based on time series, because the characteristics of 


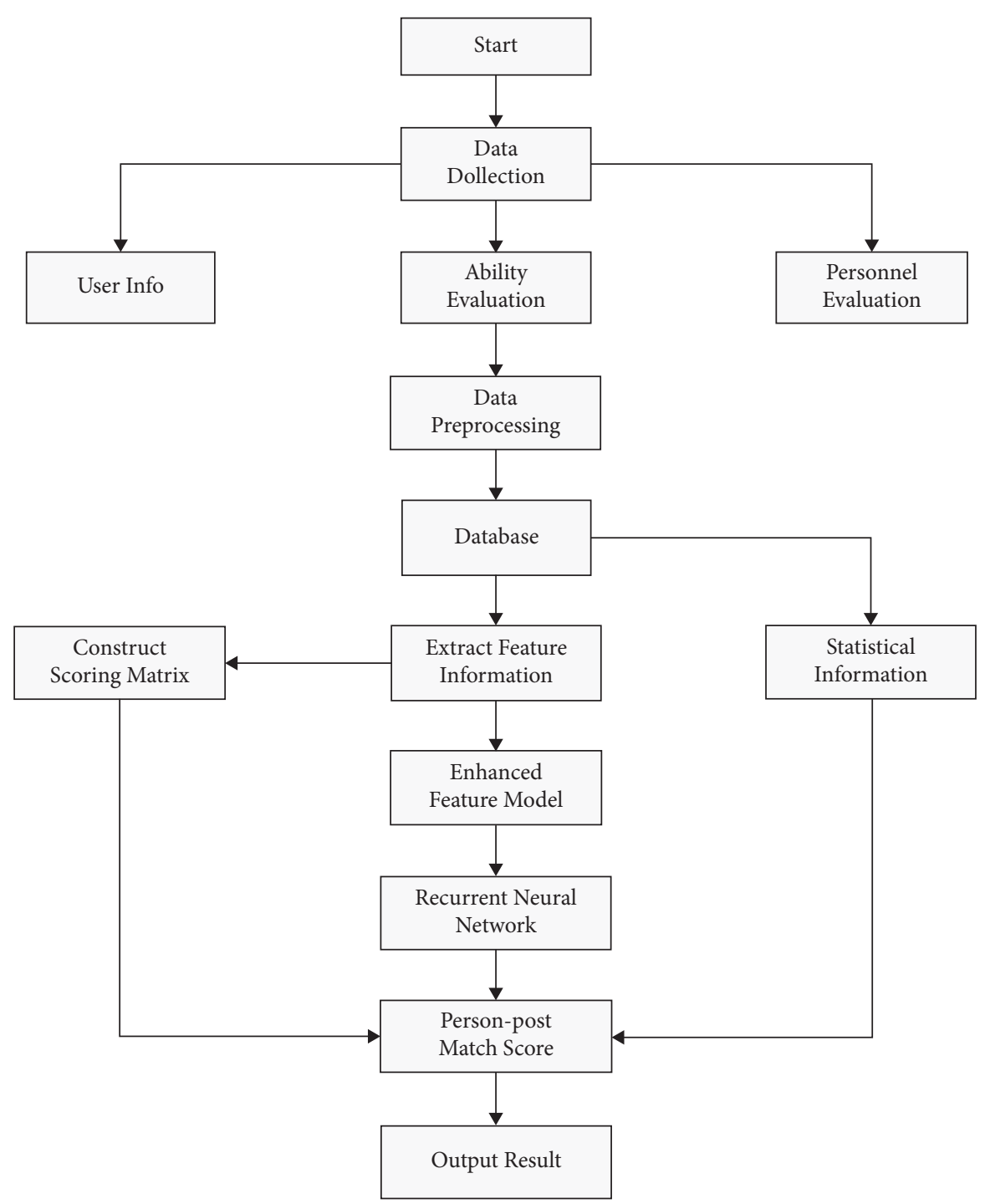

FIgURE 3: The flow diagram of the proposed algorithm.

employees cannot be judged only by the current data, so it is necessary to obtain the trend from the statistical data. Traditional personnel statistics reports have time records, so it is necessary to save the database based on time series. Therefore, in statistical analysis, by analyzing and comparing the changes of the same index in each period, we can find out the existing problems and put forward corresponding suggestions based on this.

The second step is to preprocess the original data. This step comprises the following substeps:

(1) The difference between data and information: Data and information are very different. The data we count will not be automatically transformed into directly readable information. Data is only a summary in quantitative form. Records in this quantitative form can be sorted into statistical data and become a data resource. From the perspective of statistical methods and thinking, information can be understood as an intermediate medium from data to decision-making. Whether the massive statistical data can play a role in decision-making depends on the rationality of data analysis and sorting. For decision-makers of different events, similarity of statistics, and whether they can extract effective information from the data and whether they can make the extracted information serve their own decision-making reflect the management level of different managers.

(2) Information processing of data: The process of extracting information from statistical data is the process of data processing and analysis. To transform statistical data into information serving decisionmaking, we must make timely, scientific, and systematic use of the principles, methods, and means of statistical analysis. First, we should ensure the authenticity and reliability of the original data, identify a large amount of data in the statistical data, and prevent forgery, duplication, and disclosure; 


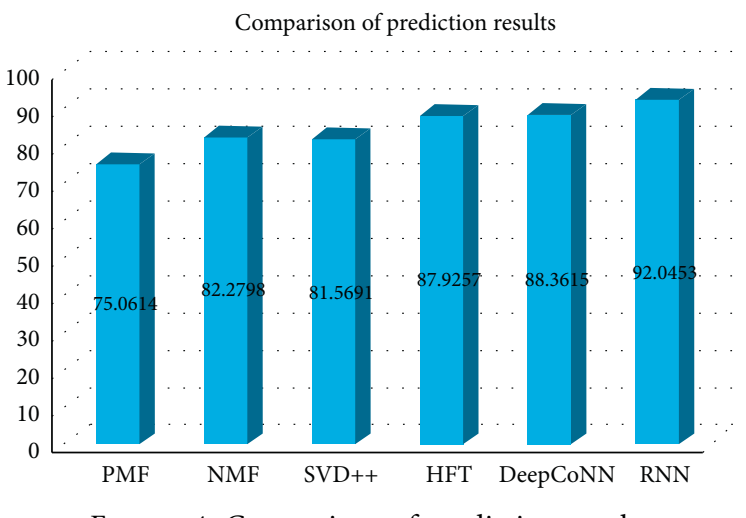

FIGURE 4: Comparison of prediction results.

secondly, strict inspection and control shall be carried out when compiling and sorting statistical data, and the consistency of index meaning and the accuracy of data calculation shall be considered. Finally, form the analysis report.

(3) Supplement and correct the data gap: Supplement and correct the problems encountered in the use of raw data to meet the requirements of management and decision-makers. The need to add and supplement new data depends on their contribution to reducing the uncertainty caused by adverse factors in the decision-making process. Usually, the decision is made when the information is incomplete or asymmetric, so the supplementary data is relatively complete.

The third step is feature enhancement.

Obtain the data from the data warehouse, identify the missing values in the data, delete the harmful data and input the missing values, then normalize or standardize the data, finally learn the data grouping results, and take the fused data as the data input of the network in the model for training.

The fourth step is the output of recommendation results.

Sort the results of the matching degree between manpower and position, output the recommendation results based on the diversity of employee characteristics and position characteristics, and then refer to the score for reasonable position allocation.

\section{Results Analysis and Discussion}

According to the above methods and algorithm flow, the ideas are sorted out before the experiment. The first is data collection. The distributed flow data collection method is used. Due to the different human data of different enterprises, the format is also different. Therefore, the manpower data must be processed in a unified format, and its data processing methods include discarding, conversion, and other operations. The second is to preprocess the original data. Group the data to get a more comprehensive understanding of the characteristics of the human resource model. At the same time, the data is saved in the data warehouse, which can support the subsequent data model training. Then, feature enhancement is carried out. Obtain data from the data warehouse and learn the data grouping results. The fused data is used as the data input of the network in the model for training. Finally, the recommendation result output. Sort the manpower and position matching results, and then refer to the score for reasonable position allocation.

The experiment will use four publicly accessible data sets from the matching of employees and positions in different enterprises to evaluate the model in this paper. These data sets cover different enterprise fields and contain parametric information of different numbers of employees, positions, and matching relationships. To evaluate the performance of model rating prediction, RNN, and five commonly used recommendation methods are applied to four data sets in different fields and compared. The five recommended models are PMF, NMF, SVD++, HFT, and DeepCoNN. The comparison of the prediction results is shown in Figure 4.

Calculate the accuracy of the above five algorithm models, respectively, take the high-quality manpower and position matching of an enterprise in a quarter as the prediction comparison model, and input the corresponding manpower characteristic parameters and position corresponding parameters. The accuracy of the prediction results is shown in the table. RNN has obvious advantages in accuracy. Comparison of RMSE of different recommendation algorithms is shown in Figure 5.

Finally, as shown in Figure 5, the RNN method in this paper is always better than all the comparison methods. Although comment information is very important in the recommendation process, different ways of using comment information will also lead to different recommendation results. The model based on the recurrent neural network proposed in this paper not only uses comments but also considers the importance of each employee's personality characteristics and professional experience, which further improves the recommendation performance. Statistical table 


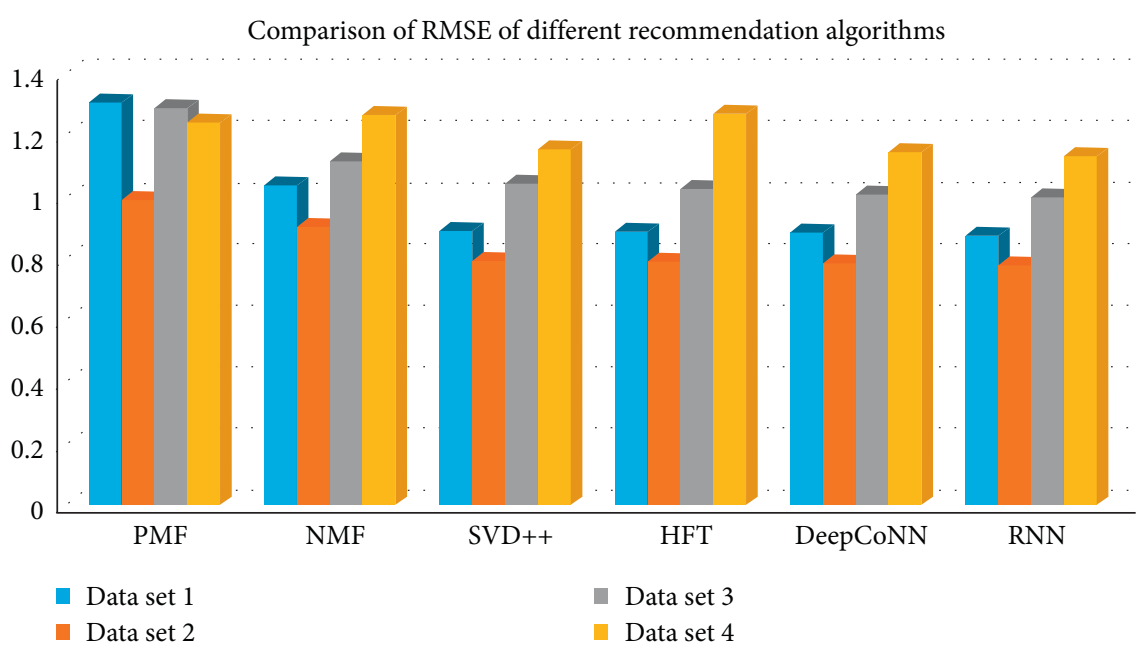

FIGURE 5: Comparison of RMSE of different recommendation algorithms.

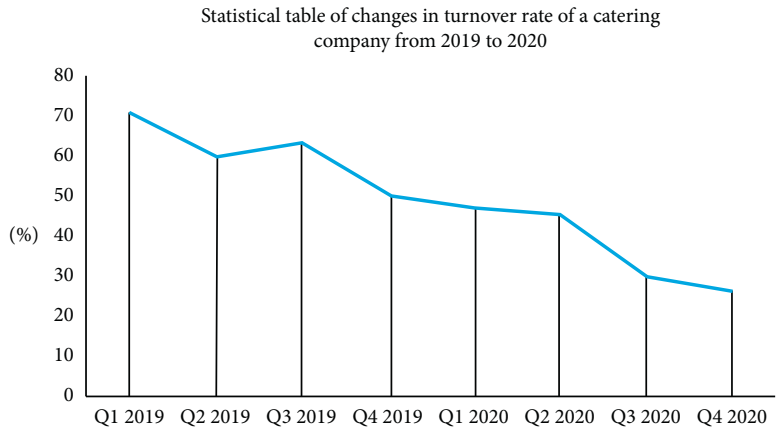

FIGURE 6: Statistical table of changes in the turnover rate of a catering company from 2019 to 2020.

of changes in turnover rate of a catering company from 2019 to 2020 is shown in Figure 6.

Finally, as shown in Figure 6, this is a statistical table of employee turnover after an enterprise optimizes the human resource allocation method. According to the trend of the chart and the previous theoretical methods, the optimization of the human resource allocation method by using a cyclic neural network algorithm can greatly reduce the turnover rate of employees and ensure the steady rise of enterprises.

\section{Conclusions and Future Work}

With the rapid development of computer technology, the deep neural network has attracted great attention in academia and industry and is widely used in image processing, engineering early warning, and so on. At present, deep learning has been adopted by recommendation system research, partly because of its ability to process sequential information. This paper mainly studies the depth recommendation model based on recurrent neural network, summarizes the existing depth recommendation models, and applies them to the allocation of human resources in enterprises. The essence of the human resource scheduling model is to analyze human resource data and calculate the matching degree between manpower and position. Afterwards, the personnel scheduling is carried out according to the matching degree score between the manpower and position, which can be potentially abstracted into a recommendation model in essence.

This paper improves the basic neural network; uses the combination of the cyclic neural network model, global model, and local model; takes the data characteristics after hierarchical operation of the model as the network output; and then realizes the data processing. By using the hierarchical model structure to build the network, we can finally realize the high-precision matching and recommendation of manpower and posts. Our future work will focus on applying the method proposed in this paper to the study of the personnel arrangement process in projects for cloud resource allocation [14, 17], considering that the working ability of personnel will directly affect the efficiency of project completion.

\section{Data Availability}

The data used to support the findings of this study are available from the corresponding author upon request.

\section{Conflicts of Interest}

The authors declare that there are no conflicts of interest. 


\section{References}

[1] Y. Xu, "How to build a strategic human resource management system for state-owned enterprises," People's Forum, vol. 4, no. 5, pp. 96-97, 2021.

[2] J. He and L. Jin, "Research on human resource quality structure recognition model based on bp neural network," Science and Management, vol. 39, no. 6, pp. 50-57, 2019.

[3] X. Gao, "Talent allocation and team building of engineering project enterprises," Human Resources, vol. 5, no. 14, pp. 6-7, 2021.

[4] X. Li, "Thoughts and measures for optimizing the allocation of talents in mining enterprises," Human Resources, vol. 5, no. 14, pp. 136-137, 2021.

[5] X. Liu, X. Yue, J. Chao, and Y. Wang, "Design and simulation of medical human resource allocation model based on recurrent neural network," Electronic Design Engineering, vol. 29, no. 11, pp. 39-43, 2021.

[6] X. Liu, X. Yang, and X. Wu, "Human resource management work allocation algorithm based on fuzzy relationship," Soft Science, vol. 17, no. 4, pp. 62-64, 2003.

[7] R. Gao, L. Jing, B. Du, Y. Yonghong, S. Chengfang, and D. Yonggang, "A point-of-interest recommendation model for location social networks integrating context and comment information," Journal of Computer Research and Development, vol. 53 , no. 4 , pp. 752-763, 2016.

[8] V. J. Hinton, N. E. Nereo, R. J. Fee, and S. E. Cyrulnik, "Social behavior problems in boys with Duchenne muscular dystrophy," Journal of Developmental and Behavioral Pediatrics, vol. 27, no. 6, pp. 470-476, 2006.

[9] T. H. Kim and P. B. Lee, "A study on improved MLP learning using RBM prior learning," Korean Society of Information Sciences, vol. 34, no. 1C, 2007.

[10] L. Edo, W. Franco, P. G. Martinsson, V. Rokhlin, and M. Tygert, "Randomized algorithms for the low-rank approximation of matrices," Proceedings of the National Academy of Sciences of the United States of America, vol. 104, no. 51, pp. 20167-20172, 2007.

[11] N. N. Le Roux, L. Roux, and Y. Bengio, "Representational power of restricted Boltzmann machines and deep belief networks," Neural Computation, vol. 20, no. 6, pp. 1631-1649, 2008.

[12] P. Hamel, S. Lemieux, Y. Bengio, and E. Douglas, "Temporal pooling and multiscale learning for automatic annotation and ranking of music audio," in Proceedings of the 12th International Society for Music Information Retrieval ConferenceIsmir, pp. 729-734, Miami, FL, USA, October, 2011.

[13] A. Oord, S. Dieleman, and B. Schrauwen, "Deep contentbased music recommendation," Advances in Neural Information Processing Systems, vol. 2, 2013.

[14] X. Du, "Research on engineering project management method based on BIM technology," Scientific Programming, vol. 2021, Article ID 7230585, 10 pages, 2021.

[15] S. Dang and W. Tang, "Real-time data scheduling of flexible job in papermaking workshop based on deep learning and improved fuzzy algorithm," Mobile Information Systems, vol. 2021, Article ID 9930740, 12 pages, 2021.

[16] H. Zhu, "Research on human resource recommendation algorithm based on machine learning," Scientific Programming, vol. 2021, Article ID 8387277, 10 pages, 2021.

[17] G. E. Hinton, "Learning multiple layers of representation," Trends in Cognitive Sciences, vol. 11, no. 10, pp. 428-434, 2007.

[18] R. Kumar, B. Verma, and S. Sunder Rastogi, "Social popularity based SVD++ recommender system," International Journal of Computer Application, vol. 87, no. 14, pp. 33-37, 2014. 